\title{
Fuzzy Multiple Objective Models For Facility Location Problems
}

\author{
Mehmet Can \\ Faculty of Arts and Social Sciences, \\ International University of Sarajevo, Paromlinska 66, \\ 71000 Sarajevo, Bosnia and Herzegovina \\ E-mail: mcan@ius.edu.ba
}

\begin{abstract}
There are a variety of efficient approaches to solve crisp multiple objective decision making problems. However in the real life the input data may not be precisely determined because of the incomplete information. This paper deals with a multi objective facility location problem using the algorithm developed by Drezner and Wesolowski.
\end{abstract}

Keywords: Fuzzy decision making, multi objective decision, fuzzy goal programming, facility location problem.

\section{INTRODUCTION}

In a standard multiple goal programming, goals and constraints are defined

\section{MULTIPLE FUZZY GOAL PROGRAMMING}

precisely. Fuzzy goal programming has the advantage of allowing for the vague aspirations of decision makers, which are quantified by some natural language rules [1-18].

To our knowledge, first R. Narasimhan [15] introduced fuzzy set theory into objective programming. Since then many achievements have been added to the literature. In the following, an approach for solving fuzzy multiple goal problems will be presented, and its application to a facility location problem will be discussed.

In a multiple goal programming problem, the optimal realization of multiple objectives is desired under a set of constraints imposed by a real life environment. If the goals and constraints are all expressed with equalities, we have a completely symmetric formulation

Find $\mathbf{x}$ such that $\mathbf{A x}=\mathbf{b}, \mathbf{x} \geq \mathbf{0}$.

Where $\mathbf{x}$ is the vector of variables, $\mathbf{b}$ is the vector of the goals and available resources, and $\mathbf{A}$ is the matrix of the coefficients. In the cases when the decision maker is not precise in goals and 
restrictions, the linguistic statements such as "around b" will be used. In this case the above crisp goal programming problem becomes

Find $\mathbf{x}$ such that

$$
\mathbf{A x}=\tilde{\mathbf{b}}, \mathbf{x} \geq \mathbf{0} \text {. }
$$

Where the fuzzy components $b_{i}$ of the fuzzy vector $\mathbf{b}$ can be represented by, for example, triangular fuzzy numbers:

$\mu_{i}(z)=\left\{\begin{array}{c}\left(z-\left(b_{i}-d_{i 1}\right)\right) / d_{i 1}, b_{i}-d_{i 1} \leq z \leq b_{i}, \\ \left(\left(b_{i}+d_{i 2}\right)-z\right) / d_{i 2}, b_{i} \leq z \leq b_{i}+d_{i 2}, \\ 0, \text { elsewhere. }\end{array}\right.$

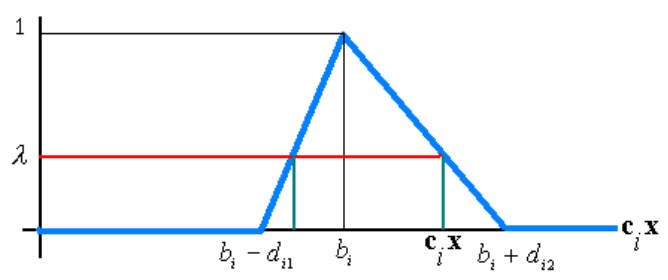

Figure 1. Fuzzy components $b_{i}$ of the fuzzy vector $\mathbf{b}$.

To have a membership number at least $\lambda$, $\mathbf{C}_{i} \mathbf{X}$ must remain in the interval

$$
b_{i}-d_{i 1}+\lambda d_{i 1} \leq \mathbf{c}_{i} \mathbf{x} \leq b_{i}+d_{i 2}+\lambda d_{i 2}
$$

that is

$$
\begin{gathered}
\left(\mathbf{c}_{i} \mathbf{x}-\left(b_{i}-d_{i 1}\right)\right) / d_{i 1} \geq \lambda, \\
\left(\left(b_{i}+d_{i 2}\right)-\mathbf{c}_{i} \mathbf{x}\right) / d_{i 2} \geq \lambda .
\end{gathered}
$$

Hence the above fuzzy goal programming problem is the maximum satisfaction problem of the fuzzy equations, and this goal can be achieved by the solution of the below crisp linear programming problem Lai, and Wang [14].
Max $\lambda$ such that for all $i$,

$$
\begin{aligned}
& \left(\mathbf{c}_{i} \mathbf{x}-\left(b_{i}-d_{i 1}\right)\right) / d_{i 1} \geq \lambda, \\
& \left(\left(b_{i}+d_{i 2}\right)-\mathbf{c}_{i} \mathbf{x}\right) / d_{i 2} \geq \lambda .
\end{aligned}
$$

\section{A FACILITY LOCATION PROBLEM}

Bhattacharya, J.R. Rao, and R.N. Twari [2] have used fuzzy goal programming to locate a single facility on a plane bounded by a convex polygon under three objectives:

i. Maximize the minimum distances,

ii. Minimize the maximum distances from the facilities to the demand points,

iii. Minimize the sum of all transport costs.

Let $P_{i}=\left(a_{i}, b_{i}\right), i=1,2, \ldots, m$ be the locations of demand points, $S=(x, y)$ is the location of the new facility, and $X$ is the set of feasible points for new facility. Then,

$\operatorname{Max} g_{1}(x, y)=\min _{\mathrm{i}}\left(\left|x-a_{i}\right|+\left|y-b_{i}\right|\right)$

$\operatorname{Min} g_{2}(x, y)=\max _{\mathrm{i}}\left(\left|x-a_{i}\right|+\left|y-b_{i}\right|\right)$

Min $g_{3}(x, y)=\Sigma_{\mathrm{i}} w_{i}\left(\left|x-a_{i}\right|+\left|y-b_{i}\right|\right)(7)$

Such that

$c_{j 1} x+c_{j 2} y \leq c_{j 3}, j=1,2, \ldots, n, \quad(x, y) \in X$.

Where $w_{i}$ 's denote the cost per unit distance between the new facility $S$ and demand points $P_{i}=\left(a_{i}, b_{i}\right)$. To describe the distances the taxicab geometry or city block distance is used since the problem is considered in an urban area. Euclidean distance could also be used.

The same problem can also be formulated as follows:

Find $S=(x, y)$ such that 


$$
\begin{aligned}
& g_{1} \geq g_{1}^{0} \\
& g_{2} \leq g_{2}^{0} \\
& g_{3} \leq g_{3}^{0} \\
& \left|x-a_{i}\right|+\left|y-b_{i}\right| \geq g_{1}, \forall i \\
& \left|x-a_{i}\right|+\left|y-b_{i}\right| \leq g_{2}, \forall i \\
& c_{j 1} x+c_{j 2} y \leq c_{j 3}, j=1,2, \ldots, n, \\
& (x, y) \in X .
\end{aligned}
$$

where $g_{1}^{0}, g_{2}^{0}, g_{3}^{0}$ are the three goals prescribed by the decision maker. One may use positive ideal solution $\left(g_{1}^{*}, g_{2}^{*}, g_{3}^{*}\right)$ to represent the goals and tolerances of fuzzy goals may be the differences of the positive and negative ideal solutions $\left(g_{1}^{-}, g_{2}^{-}, g_{3}^{-}\right)$.

Positive and negative ideal solutions are the solutions of the following problems:

$g_{1}^{*}:$ Max $g_{1}$ such that

$$
\begin{aligned}
& \left|x-a_{i}\right|+\left|y-b_{i}\right| \geq g_{1}, \forall i \\
& c_{j 1} x+c_{j 2} y \leq c_{j 3}, j=1,2, \ldots, n,(x, y) \in X .
\end{aligned}
$$

$g_{1}^{-}:$Min $g_{1}$ such that

$$
\begin{aligned}
& \left|x-a_{i}\right|+\left|y-b_{i}\right| \geq g_{1}, \forall i \\
& c_{j 1} x+c_{j 2} y \leq c_{j 3}, j=1,2, \ldots, n,(x, y) \in X .
\end{aligned}
$$

$g_{2}^{*}:$ Min $g_{2}$ such that

$$
\left|x-a_{i}\right|+\left|y-b_{i}\right| \leq g_{2}, \forall i
$$

$c_{j 1} x+c_{j 2} y \leq c_{j 3}, j=1,2, \ldots, n,(x, y) \in X$.

$g_{2}^{-}: \operatorname{Max} g_{2}$ such that

$$
\begin{gathered}
\qquad\left|x-a_{i}\right|+\left|y-b_{i}\right| \leq g_{2}, \forall i \\
c_{j 1} x+c_{j 2} y \leq c_{j 3}, j=1,2, \ldots, n,(x, y) \in X . \\
g_{3}^{*}: \operatorname{Min} g_{3} \text { such that } \\
\left|x-a_{i}\right|+\left|y-b_{i}\right| \leq g_{3}, \forall i \\
c_{j 1} x+c_{j 2} y \leq c_{j 3}, j=1,2, \ldots, n,(x, y) \in X .
\end{gathered}
$$

$g_{3}^{-}:$Max $g_{3}$ such that

$\left|x-a_{i}\right|+\left|y-b_{i}\right| \leq g_{3}, \forall i$

$c_{j 1} x+c_{j 2} y \leq c_{j 3}, j=1,2, \ldots, n,(x, y) \in X$.

Then using the values of $\left(g_{1}^{*}, g_{2}^{*}, g_{3}^{*}\right)$ and $\left(g_{1}^{-}, g_{2}^{-}, g_{3}^{-}\right)$, the fuzzy limitations for the goals $\left(\tilde{g}_{1}, \tilde{g}_{2}, \tilde{g}_{3}\right)$ are obtained as follows.

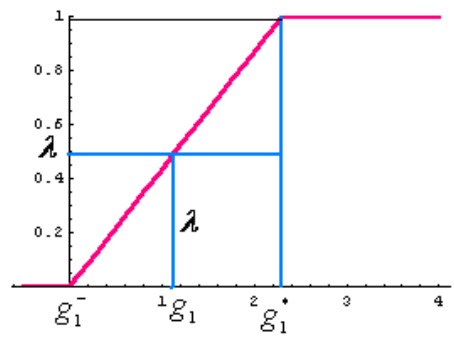

Figure 2. Fuzzy goal $\tilde{g}_{1}$.

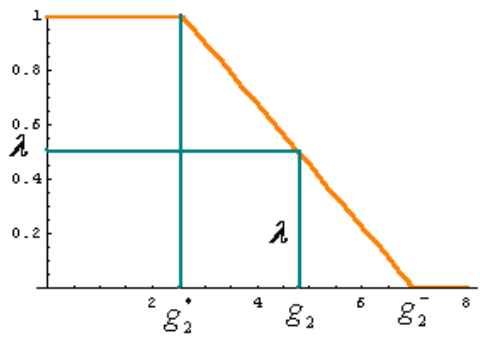

Figure 3. Fuzzy goal $\tilde{g}_{2}$. 


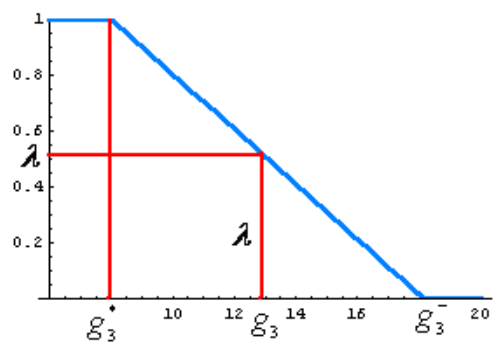

Figure 4. Fuzzy goal $\tilde{g}_{3}$.

With these fuzzy goals, problem (8) can be expressed as

Find $S=(x, y)$ Such that

$$
g_{1} \geq \tilde{g}_{1}, g_{2} \leq \tilde{g}_{2}, g_{3} \leq \tilde{g}_{3}
$$

$$
\begin{gathered}
\left|x-a_{i}\right|-\left|y-b_{i}\right| \geq g_{1}, \forall i, \\
\left|x-a_{i}\right|-\left|y-b_{i}\right| \leq g_{2}, \forall i \\
c_{j 1} x+c_{j 2} y \leq c_{j 3}, j=1,2, \ldots, n,(x, y) \in X .
\end{gathered}
$$

To transform the problem (10) into a crisp problem with only one objective, we get the $\lambda$-cuts:

Maximize $\lambda$ such that

$$
\begin{gathered}
g_{1}-g_{1}^{-} \geq \lambda\left(g_{1}^{*}-g_{1}^{-}\right) \\
-g_{2}+g_{2}^{-} \geq \lambda\left(-g_{2}^{*}+g_{2}^{-}\right), \\
-g_{3}+g_{3}^{-} \geq \lambda\left(-g_{3}^{*}+g_{3}^{-}\right) \quad, \\
\left|x-a_{i}\right|+\left|y-b_{i}\right| \geq g_{1}, \forall i \\
\left|x-a_{i}\right|+\left|y-b_{i}\right| \leq g_{2}, \forall i \\
c_{j 1} x+c_{j 2} y \leq c_{j 3}, j=1,2, \ldots, n, \quad(x, y) \in X .
\end{gathered}
$$

Hence in the feasible region $X=\{(x, y) \mid 4 x+57 \leq 20,8 x+3 y \leq 24, x, y \geq 0\}$ , the problem (16) becomes:

Maximize $\lambda$ such that

$$
\begin{aligned}
& g_{1} \geq 1.5 \lambda, g_{2} \leq 7-4 . \lambda, g_{3} \leq 35.2-16.2 \lambda \\
& x+y-g_{1} \geq c 3, x-y+g_{1} \leq c 2 \\
& x+y+g_{1} \leq c 1 \\
& x+y+g_{2} \geq c 1, x-y+g_{2} \geq c 2 \\
& x+y-g_{2} \geq c 3, x-y-g_{2} \geq c 4 \\
& 4 x+5 y \leq 20,8 x+3 y \leq 24 . x, y \geq 0 .
\end{aligned}
$$

Where

$$
\begin{aligned}
& c 1=\max _{i}\left(a_{i}+b_{i}\right), c 2=\max _{i}\left(a_{i}-b_{i}\right), \\
& c 3=\min _{i}\left(a_{i}+b_{i}\right), c 4=\min _{i}\left(a_{i}-b_{i}\right) .
\end{aligned}
$$

The solution of the above problem is found to be $x=1.696, y=2.642, \lambda=0.831$. For this optimum supply point $S=(1.31,2.23)$, one has

$$
\begin{aligned}
& g_{1}(x, y)=1.419, g_{2}(x, y)=3.458, \\
& g_{3}(x, y)=20.838
\end{aligned}
$$

\section{AN APLICATION}

Let

$$
\left\{P_{i}\right\}=\{(0,2),(2,1),(3,4)(1,3.5)(2.5,2)(2,4)(1,0)(.5, .5)\}
$$




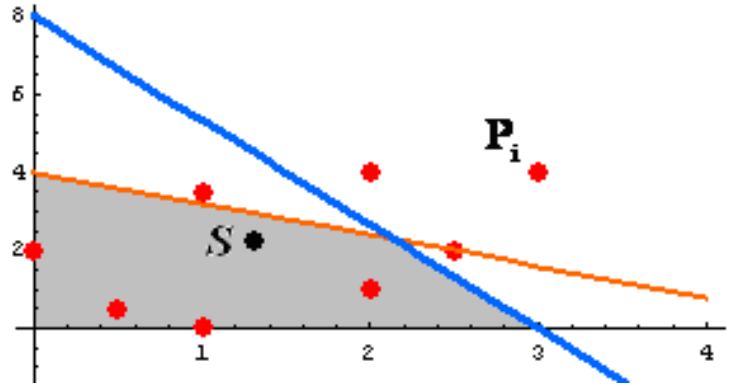

Figure 5. The three demand points $\left\{P_{i}\right\}=\left\{\begin{array}{l}(0,2),(2,1),(3,4)(1,3.5) \\ (2.5,2)(2,4)(1,0)(.5, .5)\end{array}\right\}$, and the new optimum supply point $S=(1.31,2.23)$.

\section{DISCUSSION}

In this article, to deal with and optimization problem with three fuzzy objectives, a method due to Narasimhan, $\mathrm{R}$. is used. The maximum and minimum solutions of each sub problems are found, and then using this information, goals are transformed into fuzzy inequalities. Then a crisp symmetric optimization problem is obtained by $\lambda$-cuts. The number of the demand points can be increased to represent a real world problem easily.

\section{REFERENCES}

[1]. Abo-Sinna, M.A., Multiple objective (fuzzy) dynamic programming problems: a survey and some applications, Applied Mathematics and Computation 157/3 (2004) 861-888

[2]. Bhattacharya, J.R. Rao, and R.N. Twari, Fuzzy multi-criteria facility location, Fuzzy Sets and Systems 51 (1992) 277-287.

[3]. Buckley, J.J., Multiobjective possibilistic linear programming, Fuzzy Sets and Systems, 35 (1990) 23-28.
[4]. Chanas, S., D. Kutcha, Multiobjective programming in optimization of the interval objective function-a generalized approach, European Journal of Operational Research 94 (1996) 594-598. [5]. Deb, K.,Multi-Objective Optimization using Evolutionary Algorithms, John Wiley \& Sons, England (2001).

[6]. Dey, J.K., S. Kar, and M. Maiti, An interactive method for inventory control with fuzzy lead-time and dynamic demand, European Journal of Operational Research 167 (2004) 381-397.

[7]. Eatman, J.L., and Sealey, Jr., A multiobjective linear programming model for commercial bank balance sheet management, Journal of Bank research 9 (1979) 227-236.

[8]. French, S., Interactive multiobjective programming: Its aims, applications, and demands, Journal of Operational Research Society 30 (1984) 824-837.

[9]. Hannan, E.L., On the efficiency of the product operator in fuzzy programming with multiple objectives, Fuzzy Sets and Systems 2 (1979) 259-262.

[10]. Hannan, E.L., Linear programming with multiple fuzzy goal, Fuzzy Sets and Systems 6 (1981) 235-248.

[11]. Hannan, E.L., Fuzzy decision making with multiple objective and discrete membership functions, International Journal of man-machine Studies 18 (1983) 49-54.

[12]. Hwang, C.L., S.R. Paidy, and K. Yoon, Mathematical Programming with multiple objectives: a tutorial, Computers and Operations research, 7 (1980) 5-31.

[13]. Ishibuchi, H., and H. Tanaka, Multiobjective programming in optimization of the interval objective function, European Journal of Operational Research 48 (1990) 219-225. [14]. Lai, Y.J., and Hwang, C.L. Fuzzy Multi Objective Decision Making, Springer, 2nd ed. ( 1996). 
[15]. Narasimhan, R., Goal programmimg in a fuzzy environment, Decision Sciences 11 (1980) 325-338.

[16]. Li, X., B. Zhang and H. Li, Computing efficient solutions to fuzzy multiple objective linear programming problems, Fuzzy Sets and Systems 157/10 (2006) 1328-1332.

[17]. Rommenfanger, H.,and R.Slowinski, Fuzzy linear programming with single or multiple objective functions, In Slowinski R.(Ed), Fuzzy Sets in Decision Analysis, Kuwler, Boston, (1998)

[18]. Sakawa, M., and H. Yano, Multiobjective fuzzy linear regression analysis for fuzzy input-output data, Fuzzy Sets and Systems 47 (1992) 173181. 